\title{
MACHINE LEARNING METHODS FOR SPAM E-MAIL ClassificATION
}

\author{
W.A. Awad ${ }^{1}$ and S.M. ELseuofi ${ }^{2}$ \\ ${ }^{1}$ Math.\&Comp.Sci.Dept., Science faculty, Port Said University \\ wealak@yahoo.com \\ ${ }^{2}$ Inf. System Dept.,Ras El Bar High inst. \\ Sherif.shawki@gmail.com
}

\begin{abstract}
The increasing volume of unsolicited bulk e-mail (also known as spam) has generated a need for reliable anti-spam filters. Machine learning techniques now days used to automatically filter the spam e-mail in a very successful rate. In this paper we review some of the most popular machine learning methods (Bayesian classification, k-NN, ANNs, SVMs, Artificial immune system and Rough sets) and of their applicability to the problem of spam Email classification. Descriptions of the algorithms are presented, and the comparison of their performance on the SpamAssassin spam corpus is presented.
\end{abstract}

\section{KEYWORDS}

Spam, E-mail classification, Machine learning algorithms

\section{INTRODUCTION}

Recently unsolicited commercial / bulk e-mail also known as spam, become a big trouble over the internet. Spam is waste of time, storage space and communication bandwidth. The problem of spam e-mail has been increasing for years. In recent statistics, $40 \%$ of all emails are spam which about 15.4 billion email per day and that cost internet users about $\$ 355$ million per year. Automatic e-mail filtering seems to be the most effective method for countering spam at the moment and a tight competition between spammers and spam-filtering methods is going on. Only several years ago most of the spam could be reliably dealt with by blocking e-mails coming from certain addresses or filtering out messages with certain subject lines. Spammers began to use several tricky methods to overcome the filtering methods like using random sender addresses and/or append random characters to the beginning or the end of the message subject line [11]. Knowledge engineering and machine learning are the two general approaches used in e-mail filtering. In knowledge engineering approach a set of rules has to be specified according to which emails are categorized as spam or ham. A set of such rules should be created either by the user of the filter, or by some other authority (e.g. the software company that provides a particular rule-based spam-filtering tool). By applying this method, no promising results shows because the rules must be constantly updated and maintained, which is a waste of time and it is not convenient for most users. Machine learning approach is more efficient than knowledge engineering approach; it does not require specifying any rules [4]. Instead, a set of training samples, these samples is a set of pre classified e-mail messages. A specific algorithm is then used to learn the classification rules from these e-mail messages. Machine learning approach has been widely studied and there are lots of algorithms can be used in e-mail filtering. They include Naïve Bayes, support vector machines, Neural Networks, K-nearest neighbour, Rough sets and the artificial immune system. The paper is organized as follows: section 1 is the paper introduction, section 2 summarize the related work done using various machine learning algorithms, section 3 gives a general theoretical description on the six machine learning methods 
International Journal of Computer Science \& Information Technology (IJCSIT), Vol 3, No 1, Feb 2011

we used in this study, section 4 present detailed steps of the experiment implementation and performance comparison of the six methods, finally we closed the paper with the conclusion in section 5.

\section{RELATED WORK}

There are some research work that apply machine learning methods in e-mail classification, Muhammad N. Marsono, M. Watheq El-Kharashi, Fayez Gebali[2] They demonstrated that the naïve Bayes e-mail content classification could be adapted for layer-3 processing, without the need for reassembly. Suggestions on predetecting e-mail packets on spam control middleboxes to support timely spam detection at receiving e-mail servers were presented. M. N. Marsono, M. W. El-Kharashi, and F. Gebali[1] They presented hardware architecture of na"ive Bayes inference engine for spam control using two class e-mail classification. That can classify more 117 millions features per second given a stream of probabilities as inputs. This work can be extended to investigate proactive spam handling schemes on receiving e-mail servers and spam throttling on network gateways. Y. Tang, S. Krasser, Y. He, W. Yang, D. Alperovitch [3] proposed a system that used the SVM for classification purpose, such system extract email sender behavior data based on global sending distribution, analyze them and assign a value of trust to each IP address sending email message, the Experimental results show that the SVM classifier is effective, accurate and much faster than the Random Forests (RF) Classifier. Yoo, S., Yang, Y., Lin, F., and Moon [11] developed personalized email prioritization (PEP) method that specially focus on analysis of personal social networks to capture user groups and to obtain rich features that represent the social roles from the viewpoint of particular user, as well as they developed a supervised classification framework for modeling personal priorities over email messages, and for predicting importance levels for new messages. Guzella, Mota-Santos , J.Q. Uch, and W.M. Caminhas[4] proposed an immune-inspired model, named innate and adaptive artificial immune system (IA-AIS) and applied to the problem of identification of unsolicited bulk e-mail messages (SPAM). It integrates entities analogous to macrophages, $\mathrm{B}$ and $\mathrm{T}$ lymphocytes, modeling both the innate and the adaptive immune systems. An implementation of the algorithm was capable of identifying more than $99 \%$ of legitimate or SPAM messages in particular parameter configurations. It was compared to an optimized version of the naive Bayes classifier, which have been attained extremely high correct classification rates. It has been concluded that IA-AIS has a greater ability to identify SPAM messages, although the identification of legitimate messages is not as high as that of the implemented naive Bayes classifier.

\section{MACHINE LEARNING IN E-MAIL CLASSIFICATION}

Machine learning field is a subfield from the broad field of artificial intelligence, this aims to make machines able to learn like human. Learning here means understood, observe and represent information about some statistical phenomenon. In unsupervised learning one tries to uncover hidden regularities (clusters) or to detect anomalies in the data like spam messages or network intrusion. In e-mail filtering task some features could be the bag of words or the subject line analysis. Thus, the input to e-mail classification task can be viewed as a two dimensional matrix, whose axes are the messages and the features. E-mail classification tasks are often divided into several sub-tasks. First, Data collection and representation are mostly problemspecific (i.e. e-mail messages), second, e-mail feature selection and feature reduction attempt to reduce the dimensionality (i.e. the number of features) for the remaining steps of the task. Finally, the e-mail classification phase of the process finds the actual mapping between training 
International Journal of Computer Science \& Information Technology (IJCSIT), Vol 3, No 1, Feb 2011 set and testing set. In the following section we will review some of the most popular machine learning methods.

\subsection{Naïve Bayes classifier method}

In 1998 the Naïve Bayes classifier was proposed for spam recognition. Bayesian classifier is working on the dependent events and the probability of an event occurring in the future that can be detected from the previous occurring of the same event [12]. This technique can be used to classify spam e-mails; words probabilities play the main rule here. If some words occur often in spam but not in ham, then this incoming e-mail is probably spam. Naïve bayes classifier technique has become a very popular method in mail filtering software. Bayesian filter should be trained to work effectively. Every word has certain probability of occurring in spam or ham email in its database. If the total of words probabilities exceeds a certain limit, the filter will mark the e-mail to either category. Here, only two categories are necessary: spam or ham. Almost all the statistic-based spam filters use Bayesian probability calculation to combine individual token's statistics to an overall score [1], and make filtering decision based on the score. The statistic we are mostly interested for a token $\mathrm{T}$ is its spamminess (spam rating) [10], calculated as follows:

$$
\boldsymbol{S}[\boldsymbol{T}]=\frac{\boldsymbol{C}_{\text {Spam }}(\boldsymbol{T})}{\boldsymbol{C}_{\text {Spam }}(\boldsymbol{T})+\boldsymbol{C}_{\text {Ham }}(\boldsymbol{T})}
$$

Where $\mathbf{C}_{\text {Spam }}(\mathbf{T})$ and $\mathbf{C}_{\text {Ham }}(\mathbf{T})$ are the number of spam or ham messages containing token $\mathrm{T}$, respectively. To calculate the possibility for a message $\mathrm{M}$ with tokens $\left\{\mathrm{T}_{1}, \ldots \ldots, \mathrm{T}_{\mathrm{N}}\right\}$, one needs to combine the individual token's spamminess to evaluate the overall message spamminess. A simple way to make classifications is to calculate the product of individual token's spamminess and compare it with the product of individual token's hamminess

$$
\left(\mathrm{H}[M]=\prod_{\mathrm{I}=1}^{\mathrm{N}}\left(1-\mathrm{S}\left[T_{\mathrm{I}}\right]\right)\right)
$$

The message is considered spam if the overall spamminess product $\mathbf{S}[\mathbf{M}]$ is larger than the hamminess product $\mathbf{H}[\mathbf{M}]$. The above description is used in the following algorithm [10]:

\section{Stage1. Training}

Parse each email into its constituent tokens

Generate a probability for each token $\mathrm{W}$

$$
\mathrm{S}[\mathrm{W}]=\mathrm{C}_{\mathrm{spam}}(\mathrm{W}) /\left(\mathrm{C}_{\mathrm{ham}}(\mathrm{W})+\mathrm{C}_{\mathrm{spam}}(\mathrm{W})\right)
$$

store spamminess values to a database

\section{Stage2. Filtering}

For each message $\mathrm{M}$

while (M not end) do

scan message for the next token $\mathrm{T}_{\mathrm{i}}$

query the database for spamminess $S\left(T_{i}\right)$

calculate accumulated message probabilities

$\mathrm{S}[\mathrm{M}]$ and $\mathrm{H}[\mathrm{M}]$

Calculate the overall message filtering indication by:

$\mathrm{I}[\mathrm{M}]=\mathrm{f}(\mathrm{S}[\mathrm{M}], \mathrm{H}[\mathrm{M}])$

$\mathrm{f}$ is a filter dependent function,

such as $\mathrm{I}[\mathrm{M}]=\frac{1+\mathrm{S}[\mathrm{M}]-\mathrm{H}[\mathrm{M}]}{2}$ 


$$
\begin{aligned}
& \text { if } \mathrm{I}[\mathrm{M}]>\text { threshold } \\
& \text { msg is marked as spam } \\
& \text { else } \quad \text { msg is marked as non-spam }
\end{aligned}
$$

\subsection{K-nearest neighbour classifier method}

The k-nearest neighbour $(K-\mathrm{NN})$ classifier is considered an example-based classifier, that means that the training documents are used for comparison rather than an explicit category representation, such as the category profiles used by other classifiers. As such, there is no real training phase. When a new document needs to be categorized, the $\mathrm{k}$ most similar documents (neighbours) are found and if a large enough proportion of them have been assigned to a certain category, the new document is also assigned to this category, otherwise not . Additionally, finding the nearest neighbours can be quickened using traditional indexing methods. To decide whether a message is spam or ham, we look at the class of the messages that are closest to it. The comparison between the vectors is a real time process. This is the idea of the $\mathrm{k}$ nearest neighbor algorithm:

\section{Stage1. Training}

Store the training messages.

\section{Stage2. Filtering}

Given a message $x$, determine its $k$ nearest neighbours among the messages in the training set. If there are more spam's among these neighbours, classify given message as spam. Otherwise classify it as ham.

The use here of an indexing method in order to reduce the time of comparisons which leads to an update of the sample with a complexity $O(m)$, where $\mathrm{m}$ is the sample size. As all of the training examples are stored in memory, this technique is also referred to as a memory-based classifier [6]. Another problem of the presented algorithm is that there seems to be no parameter that we could tune to reduce the number of false positives. This problem is easily solved by changing the classification rule to the following $l / k$-rule:

If $l$ or more messages among the $k$ nearest neighbours of $x$ are spam, classify $x$ as spam, otherwise classify it as legitimate mail.

The $k$ nearest neighbour rule has found wide use in general classification tasks. It is also one of the few universally consistent classification rules.

\subsection{Artificial Neural Networks classifier method}

An artificial neural network (ANN), also called simply a "Neural Network" (NN), is a computational model based on biological neural networks. It consists of an interconnected collection of artificial neurons. An artificial neural network is an adaptive system that changes its structure based on information that flows through the artificial network during a learning phase. The ANN is based on the principle of learning by example. There are, however the two classical kind of the neural networks, perceptron and the multilayer perceptron. Here we will 
International Journal of Computer Science \& Information Technology (IJCSIT), Vol 3, No 1, Feb 2011 focus on the perceptron algorithm. The idea of the perceptron is to find a linear function of the feature vector $f(x)=w^{T} x+b$ such that $f(x)>0$ for vectors of one class [2], and $f(x)<0$ for vectors of other class. Here $\mathrm{w}=\left(w_{1} w_{2, \ldots} w_{m}\right)$ is the vector of coefficients (weights) of the function, and $\mathrm{b}$ is the so-called bias. If we denote the classes by numbers +1 and -1 , we can state that we search for a decision function $d(x)=\operatorname{sign}\left(w^{T} x+b\right)$. The perceptron learning is done with an iterative algorithm. It starts with arbitrarily chosen parameters $\left(w_{0}, b_{0}\right)$ of the decision and updates them iteratively. On the $n$-th iteration of the algorithm a training sample $(x, c)$ is chosen such that the current decision function does not classify it correctly (i.e. $\left.\operatorname{sign}\left(w_{n} x+b_{n}\right) \neq c\right)$. The parameters $\left(w_{n}, b_{n}\right)$ are then updated using the rule:

$$
w_{n+1}=w_{n}+c x \quad b_{n+1}=b_{n}+c
$$

The algorithm stops when a decision function is found that correctly classifies all the training samples. The above description is used in the following algorithm [8].

\section{Stage1. Training}

Initialize $w$ and $b$ ( to random values or to 0 ).

Find a training example $(x, c)$ for which sign $\left(w^{T} x+b\right)$.

If there is no such example, then training is completed

Store the final $w$ and stop.

Otherwise go to next step

Update $(w, b): w:=w+c x, b:=b+c$. Go to previous step.

\section{Stage2. Filtering}

Given a message $\mathrm{x}$, determine its class as sign $\left(w^{T} x+b\right)$

\subsection{Support Vector Machines classifier method}

Support Vector Machines are based on the concept of decision planes that define decision boundaries. A decision plane is one that separates between a set of objects having different class memberships, the SVM modeling algorithm finds an optimal hyperplane with the maximal margin to separate two classes, which requires solving the following optimization problem.

Maximize

$$
\sum_{i=1}^{n} \alpha_{i}-1 / 2 \sum_{i, j=1}^{n} \alpha_{i} \alpha_{j} y_{i} y_{j} K\left(x_{i}, x_{j}\right)
$$

Subject to

$$
\sum_{i=1} \alpha_{i} y_{i}=0
$$

where $0 \leq \alpha \mathrm{i} \leq \mathrm{b}, \mathrm{i}=1,2, \ldots \mathrm{n}$

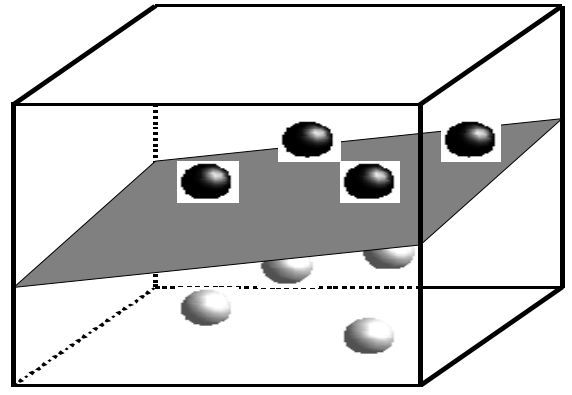

Figure 1 An SVM separating black and white points in 3 dimensions 
International Journal of Computer Science \& Information Technology (IJCSIT), Vol 3, No 1, Feb 2011 Where $\alpha_{i}$ is the weight of training sample $x_{1}$. If $\alpha_{i}>0, x_{1}$ is called a support vector $b$ is a regulation parameter used to trade-off the training accuracy and the model complexity so that a superior generalization capability can be achieved. $K$ is a kernel function, which is used to measure the similarity between two samples. A popular radial basis function $(R B F)$ kernel functions.

$$
K\left(x_{i}, x_{j}\right)=\exp \left(-\gamma\left\|x_{i}-x_{j}\right\|^{2}\right), \gamma>0
$$

After the weights are determined [9], a test sample $x$ is classified by

$$
\begin{aligned}
& y=\operatorname{Sign}\left(\sum_{i=1}^{n} \alpha_{i} y_{i} K\left(x_{i}, x_{j}\right)\right), \\
& \operatorname{Sign}(a)=\left\{\begin{array}{cc}
+1, & \text { if } a>0 \\
-1, & \text { otherwise }
\end{array}\right.
\end{aligned}
$$

To determine the values of $\langle\gamma, \mathrm{b}\rangle$, a cross validation process is usually conducted on the training dataset [3]. Cross validation is also used to estimate the generalization capability on new samples that are not in the training dataset. A k-fold cross validation randomly splits the training dataset into $k$ approximately equal-sized subsets, leaves out one subset, builds a classifier on the remaining samples, and then evaluates classification performance on the unused subset. This process is repeated $k$ times for each subset to obtain the cross validation performance over the whole training dataset. If the training dataset is large, a small subset can be used for cross validation to decrease computing costs. The following algorithm[7] can be used in the classification process.

Input : sample $x$ to classify

training set $T=\left\{\left(x_{1}, y_{1}\right),\left(x_{2}, y_{2}\right), \ldots \ldots\left(x_{n}, y_{n}\right)\right\}$;

number of nearest neighbours $k$.

Output: decision $y_{\mathrm{p}} \in\{-1,1\}$

Find $k$ sample $\left(x_{i}, y_{i}\right)$ with minimal values of $K\left(x_{i}, x_{i}\right)-2 * K\left(x_{i}, x\right)$

Train an SVM model on the $k$ selected samples

Classify $x$ using this model, get the result $y_{\mathrm{p}}$

Return $y_{\mathrm{p}}$

\subsection{Artificial Immune System classifier method}

Biological immune System has been successful at protecting the human body against a vast variety of foreign pathogens. A role of the immune system is to protect our bodies from infectious agents such as viruses, bacteria. On the surface of these agents are antigens that allow the identification of the invading agents, thus provoking an immune response Recognition in the immune system is performed by lymphocytes. Each lymphocyte expresses receptor molecules of one particular shape on its surface called antibody. An elaborate genetic mechanism involving combinatorial association of a number of gene segments underlies the construction of these receptors. The overall immune response involves three evolutionary methods: gene library, negative selection and clonal selection. In gene library, antibodies recognize antigens by the complementary properties that belong only to antigens. Thus, some knowledge of antigen properties is required to generate competent antibodies. Because of this evolutionary self- 
International Journal of Computer Science \& Information Technology (IJCSIT), Vol 3, No 1, Feb 2011 organization process, in spam management the gene libraries act as archives of information on how to detect commonly observed antigens. An important constraint that the immune has to satisfy is not to attack self cells. Negative selection eliminates inappropriate antibodies which bind to self. Clonal selection clones antibodies performing well. Thus, according to currently existing antigens, only the fittest antibodies survive. Similarly, instead of having the information about specific antigens, it organizes the fittest antibodies by interacting with the current antigens. The above description is used in the following algorithm[5]:

\section{Artificial Immune System algorithm (an email message m)}

For (each term $\mathrm{t}$ in the message) do \{

If (there exists a detector $p$, based on base

String $r$, matches with $\mathrm{t}$ ) then \{

If ( $\mathrm{m}$ is spam) then \{

Increase r's spam score by s-rate;

\} else \{

Increase r's ham score by ns-rate;

\}

\} else \{

if ( $\mathrm{m}$ is spam) then \{

if (detector $\mathrm{p}$ recognizes $\mathrm{t}$ and $\operatorname{edmf}(\mathrm{p}, \mathrm{t})>$ threshold) then \{

The differing characters are added to its corresponding entry in the library of character generalization rules;

\} else \{

A new base string $\mathrm{t}$ is added into the library of base strings;

\}

\}

\}

Decrease the age of every base string by a-rate;

\}

\subsection{Rough sets classifier method}

In 1982 Rough set (RS) theory was developed by Pawlak. Rough set has a great ability to compute the reductions of information systems. Information system might has some attributes that are irrelevant to the target concept (i.e. decision attribute), and some redundant attributes. Reduction is needed to generate simple useful knowledge from it. It is a minimal subset of condition attributes with respect to decision attributes. The Rough set scheme is provided as follows.

Step 1: With the the incoming emails, first thing we need to do is to select the most appropriate attributes to use for classification. Then the input dataset is transformed into a decision system, which is then split into the training dataset and the testing dataset. A classifier will be induced from the training dataset and applied to the testing dataset to obtain performance estimation. For training dataset, do Step 2 and Step 3.

Step 2: Because the decision system has real values attributes, Boolean reasoning algorithm should be used to finish the discretization strategies.

Step 3: Genetic algorithms should be used to get the decision rules. Then For testing dataset, continue to Step 4. 
International Journal of Computer Science \& Information Technology (IJCSIT), Vol 3, No 1, Feb 2011

Step 4: First, discretizes the testing dataset employing the same cuts computed from step 2. Then the rules generated in Step 3 are used to match every new object in testing dataset to make decision.

Let $b=0.15 \in(0,1 / 2)$ be the threshold for positive region, therefore, these $b$ - lower and $b$ upper approximations divide the whole emails in tree regions, called 0.15-positive, 0.15-boundary and 0.15-negative regions, The algorithm is described as follows:

Input : Dis_testing dataset, $R U L, b$.

/* Dis_testing dataset: Discretized testing dataset using cuts obtained from step2 and RUL - the rules generated in Step 3. Rel( ) denotes an object $\mathrm{x}$ is relevant to non-spam. CERx denotes the sum predicts number for object $x . \mathrm{b}=0.15 \in(0,1 / 2) * /$

Output : the three categories - non spam, spam and suspicious.

for $x \in D i s \_T E$ do

while $R U L(x)=0$ do

$$
\text { suspicious }=\text { suspicious } \cup\{x\} \text {; }
$$

end

Let all $r \in R U L(x)$ cast a number in favor of the non-spam class. The number of predicts a rule gets to cast is actually the membership degree based on the decision rules; $R=r \in R U L(x) \mid r$ predicts non-spam;

Estimate Rel(Dis_T E |x $x \in$ non-spam);

$\operatorname{Rel}\left(\right.$ Dis_TE $\mid x \in$ non-spam) $=\sum r \in R$ Predicts(non-spam);

Certainty $_{x}=1 /$ cer $\times \operatorname{Rel}($ Dis_T E $\mid x \in$ non-spam);

while Certainty $y_{x} \geq 1-$ b do suspicious $=$ suspicious $\cup\{x\}$;

end

spam $=$ spam $\cup\{x\} ;$

end

\section{MACHINE LEARNING METHODS PERFORMANCE}

\subsection{Experiment Implementation}

In order to test the performance of above mentioned six methods, some corpora of spam and legitimate emails had to be compiled; there are several collections of email publicly available to be used by researchers. SpamAssassin (http://spamassassin.apache.org) will be used in this experiment, which contains 6000 emails with the spam rate $37.04 \%$. Thus we have divided the corpora into training and testing sets keeping, in each such set, the same proportions of ham (legitimate) and spam messages as in the original example set. Each training set produced contained $62.96 \%$ of the original set; while each test set contain $37.04 \%$ as Table 1 . 
International Journal of Computer Science \& Information Technology (IJCSIT), Vol 3, No 1, Feb 2011

Table 1. Corpora of Spam and Ham Messages

\begin{tabular}{|l|l|l|}
\hline Message collection & Training Set & Testing Set \\
\hline Ham Messages & 2378 & 1400 \\
\hline Spam Messages & 1398 & 824 \\
\hline Total Messages & 3776 & 2224 \\
\hline
\end{tabular}

In addition to the body message of an email, an email has another part called the header. The job of the header is to store information about the message and it contains many fields like the field (From) and (Subject), we decided to divide the email into 3 different parts. The first part is the (Subject) that can be considered as the most important part in the email, it noticed that most of the new incoming emails have descriptive Subjects that can be used to clearly identify whether that email is Spam or Ham. The second part is (From) which is the person that taking the responsibility of the message, this field we store it in a database and use it after the decision of the classifier has been taken, that is the way to compare the field (From) stored in the database to the field (From) in the new incoming email, if they are the same so the decision of the new incoming email is Spam. The (Body) is the third part which is the main part of the message. Furthermore we applied two procedures in the preprocessing stage. Stopping is employed to remove common word. Case-change is employed to change the (Body) into small letters. The experiment is performed with the most frequent words in spam email; we select 100 of them as features.

\subsection{Detailed algorithm steps}

\section{Step 1: Email preprocessing}

The content of email is received through our software, the information is extracted then as mentioned above, then the information (Feature) extracted is saved into a corresponding database. Every message was converted to a feature vector with 21700 attributes (this is approximately the number of different words in all the messages of the corpus). An attribute $n$ was set to 1 if the corresponding word was present in a message and to 0 otherwise. This feature extraction scheme was used for all the algorithms.

\section{Step 2: Description of the feature extracted}

Feature extraction module extract the spam text and the ham text, then produce feature dictionary and feature vectors as input of the selected algorithm, the function of feature extraction is to train and test the classifier [9]. For the train part, this module account frequency of words in the email text, we take words which the time of appearance is more than three times as the feature word of this class. And denote every email in training as a feature vector.

\section{Step 3: Spam classification}

Through the steps above, we take standard classification email documents as training document, pretreatment of email, extract useful information, save into text documents according to fix format, split the whole document to words, extract the feature vector of spam document and translate into the form of vector of fix format. We look for the optimal classification using the selected algorithm which is constructed using the feature vector of spam documents.

\section{Step 4: Performance evaluation}

In order to test the performance of above mentioned six methods, we used the most popular evaluation methods used by the spam filtering researchers. Spam Precision (SP), Spam Recall (SR), Accuracy (A). Spam Precision (SP) is the number of relevant documents identified as a

$$
\mathrm{SP}=\frac{\text { \# of Spam Correctly Classified }}{\text { Total \# of messages classifies as spam }}=\frac{\mathrm{N}_{\mathrm{snam} \rightarrow \text { snam }}}{\mathrm{N}_{\text {snam } \rightarrow \text { snam }}+\mathrm{N}_{\mathrm{ham} \rightarrow \text { snam }}}
$$


International Journal of Computer Science \& Information Technology (IJCSIT), Vol 3, No 1, Feb 2011 percentage of all documents identified; this shows the noise that filter presents to the user (i.e. how many of the messages classified as spam will actually be spam)

Spam Recall (SR) is the percentage of all spam emails that are correctly classified as spam.

$$
\mathrm{SR}=\frac{\# \text { of Spam Correctly Classified }}{\text { Total \# of messages }} \quad=\quad \frac{\mathrm{N}_{\text {spam } \rightarrow \text { spam }}}{\mathrm{N}_{\text {spam } \rightarrow \text { spam }}+\mathrm{N}_{\text {spam } \rightarrow \text { ham }}}
$$

Accuracy (A) is the percentage of all emails that are correctly categorized

$$
A=\frac{\# \text { of e-mails correctly categorized }}{\text { Total \# of e-mails }}=\frac{N_{\text {ham } \rightarrow \text { ham }}+N_{\text {spam } \rightarrow \text { spam }}}{N_{\text {ham }}+N_{\text {spam }}}
$$

Where $\mathrm{N}_{\text {ham } \rightarrow \text { ham }}$ and $\mathrm{N}_{\text {spam } \rightarrow \text { spam }}$ are the number of messages that have been correctly classified to the legitimate email and Spam email respectively; $\mathrm{N}_{\mathrm{ham} \rightarrow \text { spam }}$ and $\mathrm{N}_{\text {spam } \rightarrow \text { ham }}$ are the number of legitimate and spam messages that have been misclassified; $\mathrm{N}_{\text {ham }}$ and $\mathrm{N}_{\text {spam }}$ are the total number of legitimate and spam messages to be classified.

\subsection{Performance Comparison}

We summarize the performance result of the six machine learning methods in term of spam recall, precision and accuracy. Table 1 and Figure 2 summarize the results of the six classifiers by selecting the top 100 features (the most relevant word). In term of accuracy we can find that the Naïve bayes method is the most accurate while the artificial immune System and the $\boldsymbol{k}$ nearest neighbour give us approximately the same lower percentage, while in term of spam precision we can find that the Nä̈ve bayes method has the highest precision among the six algorithms while the $\boldsymbol{k}$-nearest neighbour has the worst precision percentage and surprisingly the rough sets method has a very competitive percent, and finally we can find that the recall is the less percentage among the six classifiers while the Nä̈ve bayes still has the highest performance but considered low when compared to precision and accuracy while the rough sets has the worst performance.

Table 2. Performance of six machine learning algorithms by selecting top 100 features

\begin{tabular}{|c|c|c|c|}
\hline Algorithm & $\begin{array}{c}\text { Spam Recall } \\
(\mathbf{\%})\end{array}$ & $\begin{array}{c}\text { Spam Precision } \\
(\mathbf{\%})\end{array}$ & $\begin{array}{c}\text { Accuracy } \\
(\mathbf{\%})\end{array}$ \\
\hline NB & 98.46 & 99.66 & 99.46 \\
\hline SVM & 95.00 & 93.12 & 96.90 \\
\hline KNN & 97.14 & 87.00 & 96.20 \\
\hline NN & 96.92 & 96.02 & 96.83 \\
\hline AIS & 93.68 & 97.75 & 96.23 \\
\hline RS & 92.26 & 98.70 & 97.42 \\
\hline
\end{tabular}


International Journal of Computer Science \& Information Technology (IJCSIT), Vol 3, No 1, Feb 2011

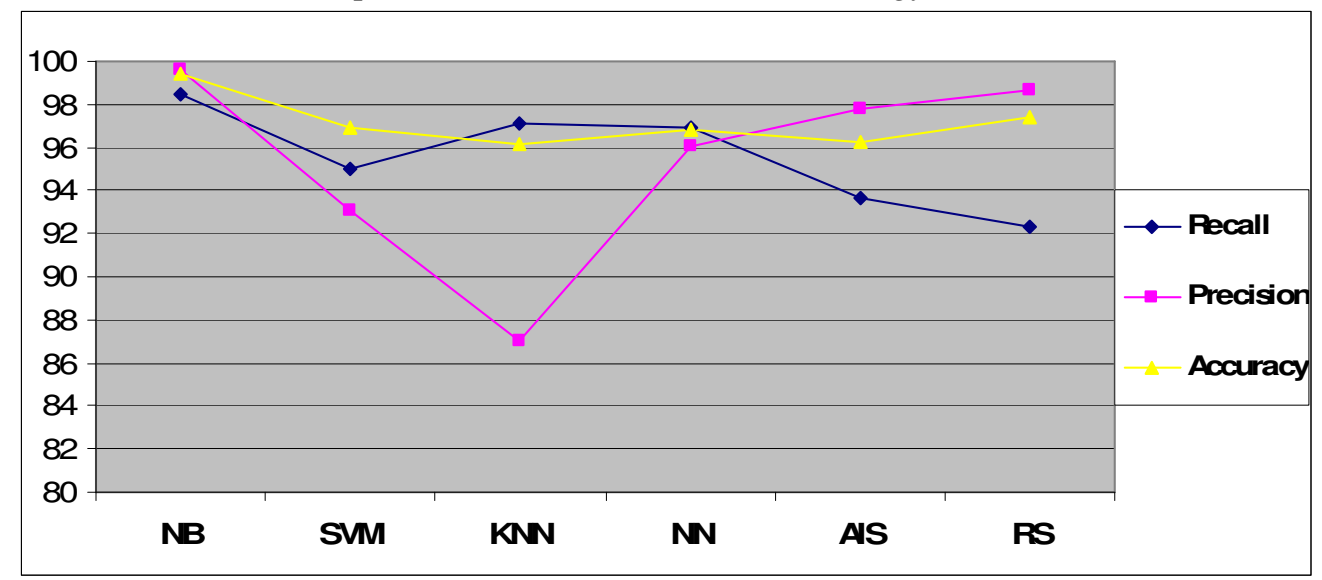

Figure 1. Spam Recall, Spam Precision and Accuracy curves of six classifiers

The performance of the $\boldsymbol{k}$-nearest neighbour classifier appeared to be nearly independent of the value of $\mathrm{k}$. In general it was poor, and it has the worst precision percentage. The performance of the neural networks was the most simple and fastest algorithm, while the rough sets method is the most complicated and has to be hybrid with the genetics algorithm to get the decision rules. Artificial immune system surprisingly give a very satisfying results which give us a hope to get a better performance when it hybrid with the rough sets method.

\section{Conclusion}

In this paper we review some of the most popular machine learning methods and of their applicability to the problem of spam e-mail classification. Descriptions of the algorithms are presented, and the comparison of their performance on the SpamAssassin spam corpus is presented, the experiment showing a very promising results specially in the algorithms that is not popular in the commercial e-mail filtering packages, spam recall percentage in the six methods has the less value among the precision and the accuracy values, while in term of accuracy we can find that the Naïve bayes and rough sets methods has a very satisfying performance among the other methods, more research has to be done to escalate the performance of the Naïve bayes and Artificial immune system either by hybrid system or by resolve the feature dependence issue in the naïve bayes classifier, or hybrid the Immune by rough sets. Finally hybrid systems look to be the most efficient way to generate a successful anti spam filter nowadays.

\section{REFERENCES}

[1] M. N. Marsono, M. W. El-Kharashi, and F. Gebali, "Binary LNS-based naïve Bayes inference engine for spam control: Noise analysis and FPGA synthesis", IET Computers \& Digital Techniques, 2008

[2] Muhammad N. Marsono, M. Watheq El-Kharashi, Fayez Gebali "Targeting spam control on middleboxes: Spam detection based on layer-3 e-mail content classification" Elsevier Computer Networks, 2009

[3] Yuchun Tang, Sven Krasser, Yuanchen He, Weilai Yang, Dmitri Alperovitch "Support Vector Machines and Random Forests Modeling for Spam Senders Behavior Analysis" IEEE GLOBECOM, 2008 
International Journal of Computer Science \& Information Technology (IJCSIT), Vol 3, No 1, Feb 2011

[4] Guzella, T. S. and Caminhas, W. M. "A review of machine learning approaches to Spam filtering." Expert Syst. Appl., 2009

[5] Wu, C. "Behavior-based spam detection using a hybrid method of rule-based techniques and neural networks” Expert Syst., 2009

[6] Khorsi. "An overview of content-based spam filtering techniques”, Informatica, 2007

[7] Hao Zhang, Alexander C. Berg, Michael Maire, and Jitendra Malic. "SVM-KNN: Discriminative nearest neighbour classification for visual category recognition", IEEE Computer Society Conference on Computer Vision and Pattern Recognition, 2006

[8] Carpinteiro, O. A. S., Lima, I., Assis, J. M. C., de Souza, A. C. Z., Moreira, E. M., \& Pinheiro, C. A. M. "A neural model in anti-spam systems.", Lecture notes in computer science.Berlin, Springer, 2006

[9] El-Sayed M. El-Alfy, Radwan E. Abdel-Aal "Using GMDH-based networks for improved spam detection and email feature analysis"Applied Soft Computing, Volume 11, Issue 1, January 2011

[10] Li, K. and Zhong, Z., "Fast statistical spam filter by approximate classifications", In Proceedings of the Joint international Conference on Measurement and Modeling of Computer Systems. Saint Malo, France, 2006

[11] Cormack, Gordon. Smucker, Mark. Clarke, Charles " Efficient and effective spam filtering and re-ranking for large web datasets" Information Retrieval, Springer Netherlands. January 2011

[12] Almeida,tiago. Almeida, Jurandy.Yamakami, Akebo " Spam filtering: how the dimensionality reduction affects the accuracy of Naive Bayes classifiers" Journal of Internet Services and Applications, Springer London , February 2011

[13] Yoo, S., Yang, Y., Lin, F., and Moon, I. "Mining social networks for personalized email prioritization". In Proceedings of the 15th ACM SIGKDD international Conference on Knowledge Discovery and Data Mining (Paris, France), June 28 - July 01, 2009

\section{Authors}

\begin{tabular}{|c|c|}
\hline & \\
\hline W.A. Awad & S.M. ELseuofi \\
\hline Math.\&Comp.Sci.Dept., Science faculty, & Inf. System Dept.,Ras El Bar High inst. \\
\hline Port Saied University & Sherif.shawki@gmail.com \\
\hline
\end{tabular}

